\title{
MODEL-FOLLOWING ADAPTIVE EQUALIZER
}

Bertran, E.,. Jaumejoan, $X$.

E.T.S.Enginyers de Telecomunicació - U.P.C.

P.O. BOX: 30.002 . 08080-Barcelona.

ABSTRACT: A new methodology for adaptive equalizers design, that could be considered as a kind of generalized and frequency selective Automatic Gain Control (AGC) circuits, is presented. This method is based on model reference adaptive systems (MRAS), and has been obtained by applying modern Control Theories to Communication Systems [5]. Apart from the robustness, its main advantages are that a feed-back channel in not required, and that it's not based on iterative or recursive algorithms (not convergence problems). Analytical and simulation results are presented.

\section{1.- Introduction.}

Communications systems through turbulent or reflecting media have the problem of the fluctuations in the received signal level. During last years several adaptive control strategies have been proposed in order to compensate channel variations such as fadings. In this paper a study of an hyperstable adaptive system is presented, which constitutes an alternative to the adaptive ones based on variable rate transmission [1] and to adaptive systems that require an identification of the channel characteristics (by using isolated test pulses or random sequences) in order to find the optimal set of tap gains after certain number of iterations [2][4]. The adaptation method here proposed is not based on Wiener or Kalman structures, and although its applications are similar than the Widrow's Adaptive Interference Canceler [7] [3] [2], the obtained adaptive law is easy to implement if the communication system has a carrier pilot or if a reference cosine wave is send.

Non-linearities or parametric variations in the channel or in the first stages of the receiver are considered in the design. The main advantages are that a feed-back channel is not required, and that it's not based on iterative algorithms (as the classical gradient-type one). Neither the tap gain adjusting algorithm convergence nor the number of iterations are a problem for the proposed adaptive system

The basical idea is well-known: to nullify the error betwcen the received carrier (or reference input) and a signal reconstructed by an oscillator (which could be synchronized with the transmitter carrier oscillator, like in several homodine receptors). The methodology to design a control system capable to nullify this error by using the hyperstability theory is presented in the paper. If the transmitted signal is narrow-band (high carrier frequencies), it's possible to take the habitual assumption that carrier variations are an indicator of the transmitted signal ones. This supposition is studied by computer simulation.

Finally some technological aspects to implement the adaptive system are presented.

\section{2.- System structure.}

The proposed method is based on the scheme of figure 1 . The adaptation system is devoted to nullify the error $e(t)$ between the received carrier $x_{c}(t)$ and the locally generated reference one, $x_{m}(t)$. It could be considered as a kind of synchronous demodulator where the received carrier is continuously compared with the expected one in order to correct alterations in the transmission (module and phase).

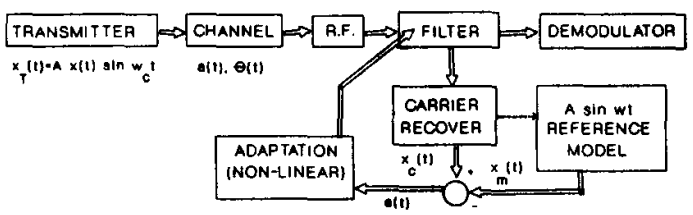

FIG.1.- System structure

3.- Design of the adaptation system.

The adaptation method described above can be modeled according to figure 2, where the reference model imposes the desired behaviour of the set channel - RF stage and filter.

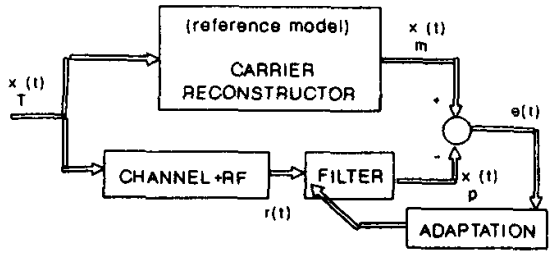

FIG.2.- Adaptation system model.

According to Popov Hyperstability Theory [6], if the model is decomposed in a linear part $(H(s))$ and a non-linear one $(M(s)$, the overall system will be asymptotically hyperstable if the linear part is real and strictly positive and the non-linear one fulfills the passivity relation:

$$
\eta(0, t)=\int \gamma^{\top} p d t \geq-\gamma_{0}^{2}
$$


being $\gamma_{0}$ an arbitrary constant, $\gamma$ the input and $\rho$ the output of the non-linear part.

Although the order of the design model implies no restrictions to the application of the method, we'll formulate the adaptation system as a second order one. Then, the selected expression for the filter is:

$$
\frac{X_{p}(s)}{R(s)}=\frac{\hat{A}_{0}(e, s)\left[1+A_{1} s+A_{2} s^{2}\right]}{1+B_{1} s+B_{2} s^{2}}
$$

being $\hat{A}_{0}(e, s)$ a function to be adjusted in order to assure that $e(t)$ tends to zero. By defining the error $e(t)$ as: $e(t)$ $=x_{m}(t) \cdot x_{p}(t)$ or, in the s-domain:

$E(s)=X_{m}(s)-\hat{A}_{0}(e, s) R(s) \frac{1+A_{1} s+A_{2} s^{2}}{1+B_{1} s+B_{2} s^{2}}$

and choosing a control law proportional plus integrative (in order to avoid steady-state errors):

$\hat{A}_{0}(e, t)=\int_{0}^{t} \psi_{1}^{0}(e, t, \tau) d \tau+\psi_{2}^{0}(e, t)+\hat{A}_{0}(0)$

the design of figure 3 , where $D(s)$ is a function to be determined (if necessary) in order to have a linear part strictly positive, is obtained.

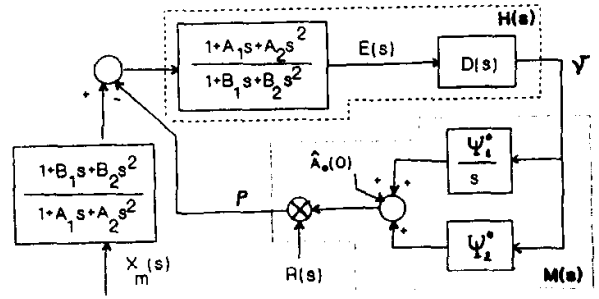

FIG.3.- Design model

Rewriting (1) as:

$\int_{0}^{t} R \gamma\left[\int_{0}^{t} \Psi_{1}^{0}(\gamma, t, \tau) d \tau+\Psi_{2}^{0}(\gamma, t)+\hat{A}_{0}(0)\right] d t>-\gamma_{0}^{2}$

and decomposing this integral in two parts [6], the following expressions for $\psi_{1}^{0}$ and $\psi_{2}^{0}$ are obtained:

$$
\begin{array}{ll}
\Psi_{1}^{0}=k_{1} R \gamma, & \text { being } k_{1}>0 \\
\psi_{2}^{0}=k_{2} R \gamma, & \text { being } k_{2}>0
\end{array}
$$

On the othei hand, the linear part (fig.3),

$$
H(s)=\frac{D(s)\left(1+A_{1} s+A_{2} s^{2}\right)}{1+B_{1} s+B_{2} s^{2}}
$$

must be a strictly positive real function (hyperstability condition), Choosing $D(s)=1$, it's obtained:

$\operatorname{Re}[H(j w)]=\frac{1-\left(A_{2}+B_{2}+A_{1} B_{1}\right) w^{2}+A_{2} B_{1} w^{4}}{\left(1-B_{2} w^{2}\right)^{2}+B_{1} w^{2}}>0, \quad \forall w$

This last equation implies that:

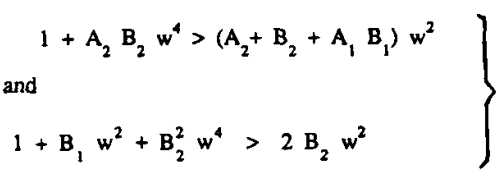

or

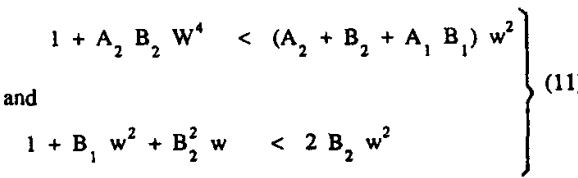

that means a bound of the adaptation system bandwidth (w max.). If it is not enough, another expression for $D(s)$ can be tried out.

The inequalities (6) and (7) are related with an atractor in the state space. Taking a large inequality the attractor has a big surface, that means a good robustness of the overall system in front of channel variations or noises. The cost of taking large inequalities is physical implementation. Also, a big attractor could represent a slow motion ( $e(t)$ tends to zero slowly).

\section{4.- Simulation results.}

According to results of section 3 , the physical model is shown in figure 4. This bas been the model used in time-simulations.

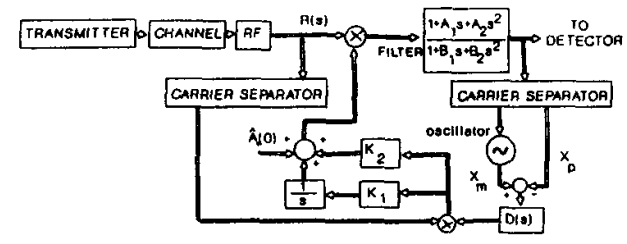

FIG.4.- Overall system.

All the simulation results are related to a normalized frequency $\Omega=w / w_{0}$ (time dilation). Figure 5 shows some results obtained with a modulating signal of $\Omega=1$, carrier frequency with $\Omega=5$ and a modulation index, $m=0,5$. Channel has been modeled as a second order low-pass filter with unitary gain and $\xi=1, \Omega_{0}=5$, Adaptation system parameters have been: $\hat{A}_{0}(0)=0, K_{1}=40, K_{2}=400, A_{1}=A_{2}=$ 
$0, B_{1}=42 / 900, B_{2}=1 / 900$. Carrier separator is based on a second order band-pass filter with $\xi=0.3$ and $\Omega_{0}=5$.

Although the results of figure 5 , the performances are not good in ASK simulations. By using a more selective carrier separator, (crystal or PLL based), the results are significatively improved. Figure 6 shows time responses of the same system when a good carrier separator is used. In this case modulating signal has a band-width of $\Omega=3$, and the carrier frequency is $\Omega_{0}=10$. Channel parameters are $\xi=$ 0,7 , gain $=0,7$ and $\Omega_{0}=30$.

Figure 7 shows the same results when channel varies to $\xi$ $=0.35$, gain $=0.7$ and $\Omega_{0}=6$. Spectra comparison (obtained from a FFT of time responses) when channel is $\xi=0.2$, gain $=0.7$ and $\Omega_{0}=20$ is shown in figure 8 .
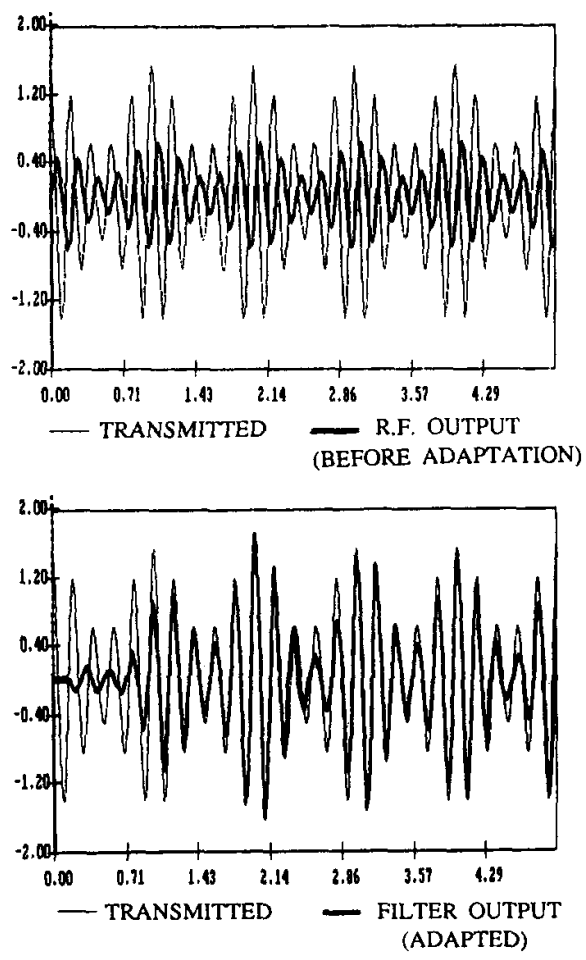

FIG.5.- Time simulations with a low selective carrier separator.

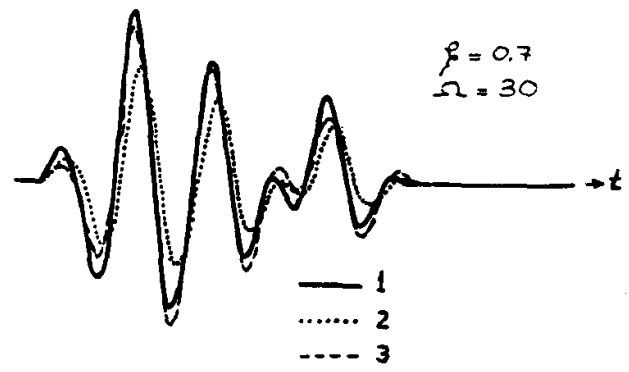

FIG.6. Time simulations with an improved carrier separator. ( I: transmitted signal. 2: received without adaptation. 3: received with adaptation).

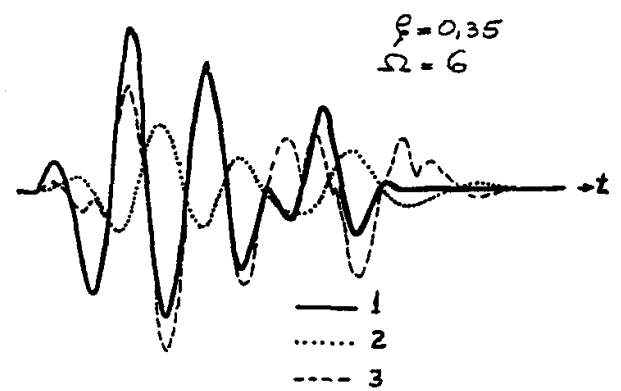

FIG.7.- Responses with a bad channel. I I: transmitted signal. 2: received without adaptation. 3: received with adaptation ).

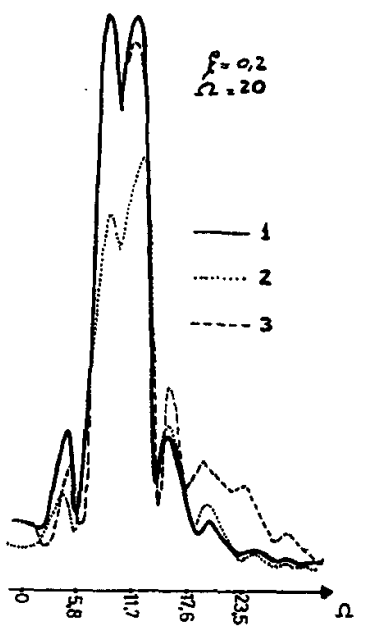

FIG.8.- Spectra comparison.( 1: transmitted signal. 2 received withous adaptation. 3: received with adaptation ). 


\section{References:}

[1] Cavers, J.K.; Lee, S.K. A Simple Buffer Control for Variable-Rate Communication systems. IEEE trans. on Communic. Vol. COM-24. September 1976.

[2] Cowan, C.F.N.; Grant, P.M. Adaptive filters. Prentice-Hall, 1985

[3] Glover, J.R. Adaptive Noise Canceling Applied to Sinusoidal Interferences. IEEE trans. on Acoust. Speech and Signal Process. Vol ASSP.25. December 1977.

[4] Hawksford, M.J.; Rezaee, N. Adaptive Mean-Square-Error Transversal Equaliser. IEE proc. Vol 128,Pt,F, n.5. Oct.1981

[5] Landau. Y.D. A Feedback Approach to Adaptive Filtering. IEEE trans. on Information Theory, Vol IT-30.n 2. March 1984

[6] Landau, Y.D. Adaptive Control. Marcel - Dekker, 1979.

[7] Widrow, B.; Sterns, S.D. Adaptive Filtering Processing. Prentice-Hall, 1985. 